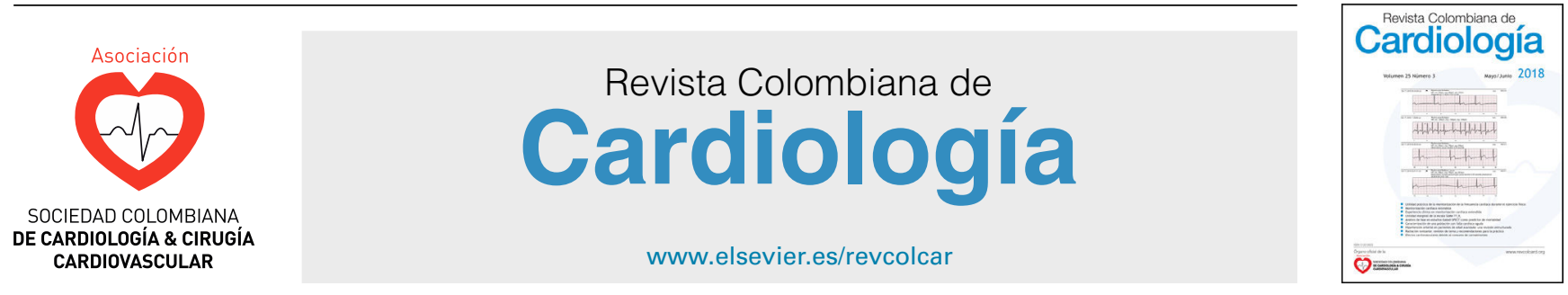

CARDIOLOGÍA DEL ADULTO - PRESENTACIÓN DE CASOS

\title{
Disección espontánea de arterias coronarias en una mujer embarazada
}

\section{Leidy Tamayo Zabala ${ }^{\mathrm{a}, *}$, Catalina Gallego Muñoz ${ }^{\mathrm{b}}$, Luis Alfonso Fajardo Andrade ${ }^{\mathrm{b}}$ y Clara Inés Saldarriaga Giraldo ${ }^{\text {b }}$}

\author{
${ }^{a}$ Medicina Interna Universidad Pontificia Bolivariana, Medellín, Colombia \\ b Clínica CardiovID, Medellín, Colombia
}

Recibido el 8 de octubre de 2018; aceptado el 26 de marzo de 2019

Disponible en Internet el 10 de julio de 2019

\section{PALABRAS CLAVE \\ Disección de las arterias coronarias; Embarazo; Síndrome coronario agudo}

\section{KEYWORDS}

Coronary artery

dissection;

Pregnancy;

Acute coronary

syndrome

\begin{abstract}
Resumen La disección espontánea de las arterias coronarias representa entre 0,2 al 4\% de las causas de síndrome coronario agudo, y es más común en mujeres jóvenes, especialmente en estado de embarazo, en cuyo caso el riesgo es tres a cuatro veces mayor. Usualmente, afecta la arteria coronaria izquierda pero también puede haber compromiso de múltiples vasos. Los pacientes que la presentan no tienen los factores de riesgo clásicos descritos de la enfermedad coronaria, como diabetes mellitus, hipertensión o dislipidemia. En las gestantes se puede presentar en cualquier trimestre del embarazo y hasta varios meses después del parto, pero es más común en el último trimestre del embarazo y en las primeras seis semanas posparto.

El diagnóstico de disección de arteria coronaria es difícil; el estándar de oro es la coronariografía con ecografía intravascular o la tomografía de coherencia óptica; esta última no se encuentra ampliamente disponible. Además, existe controversia en cuanto a la decisión de manejo médico o manejo invasivo.

(C) 2019 Sociedad Colombiana de Cardiología y Cirugía Cardiovascular. Publicado por Elsevier España, S.L.U. Este es un artículo Open Access bajo la licencia CC BY-NC-ND (http:// creativecommons.org/licenses/by-nc-nd/4.0/).
\end{abstract}

\section{Spontaneous coronary artery dissection in a pregnant woman}

Abstract Spontaneous coronary artery dissection accounts for between $0.2 \%$ and $4 \%$ of the causes of acute coronary syndrome. It is more common in young women, particularly during pregnancy, when the risk is three to four times higher. It usually involves the left coronary, but it can also compromise several vessels. The patients that present with one do not have the classic risk factors described for coronary disease, such as diabetes mellitus, hypertension, or dyslipidaemia. Although it can present in any trimester of the pregnancy, and up to several months after delivery, it is more common in the last trimester of pregnancy, and in the first six months post-partum.

\footnotetext{
* Autor para correspondencia.

Correo electrónico: tamayoleidyz@gmail.com (L. Tamayo Zabala).
} 
The diagnosis of coronary artery dissection is difficult. Although the reference method is coronary angiography with intravascular ultrasound or optical coherence computed tomography, these are not widely available, as well as there being controversy as regards the decision of medical management or invasive management.

(c) 2019 Sociedad Colombiana de Cardiología y Cirugía Cardiovascular. Published by Elsevier España, S.L.U. This is an open access article under the CC BY-NC-ND license (http:// creativecommons.org/licenses/by-nc-nd/4.0/).

\section{Introducción}

El embarazo produce grandes cambios anatómicos y fisiológicos a fin de responder al aumento en las demandas que permiten el desarrollo y el crecimiento del feto. En tal sentido, el sistema cardiovascular es uno de los que más presenta adaptaciones, con lo cual aumenta el riesgo de complicaciones o de empeorar enfermedades subyacentes. El $15 \%$ de las muertes en mujeres embarazadas es causado por enfermedad cardiovascular, en tanto que los casos correspondientes a síndrome coronario agudo se atribuyen por causas diferentes a la enfermedad ateroesclerótica como la disección espontánea de las arterias coronarias, la tromboembolia sin ateroesclerosis y el vasoespasmo ${ }^{1}$.

Aunque la disección espontánea de las arterias coronarias sólo corresponde al $4 \%$ de los síndromes coronarios agudos, su frecuencia aumenta en las mujeres embarazadas, quizás debido al incremento en los niveles de estrógeno y progesterona que producen variaciones en la pared de los vasos y a los cambios hemodinámicos del tercer trimestre del embarazo ${ }^{2}$. Su presentación atípica y baja frecuencia obligan a realizar un enfoque diagnóstico adecuado en la embarazada con dolor torácico y tener una alta sospecha de esta posible entidad para disminuir así la morbimortalidad.

A continuación se describe el caso de una paciente que en su período posparto presentó falla cardíaca de origen isquémico, sin evidencia de enfermedad coronaria en la coronariografía, pero con hallazgos sugestivos de disección espontánea de las arterias coronarias.

\section{Caso}

Primigestante de 36 años, natural y residente en Manizales, sin antecedentes personales de importancia, llevada el 12 de septiembre de 2017 a cesárea urgente por preeclampsia grave a las 37 semanas y 3 días de gestación, sin complicaciones. Fue dada de alta a los 7 días posparto junto a su recién nacido.

Nueve días después del alta presentó dolor torácico opresivo, no irradiado, de inicio súbito, disnea de pequeños esfuerzos, diaforesis y un episodio de síncope. Ingresó al servicio de urgencias, donde reportaron una taquicardia ventricular sostenida, con posterior deterioro, inestabilidad y choque cardiogénico. Requirió soporte ventilatorio, vasopresor e implantación de balón de contrapulsación intraaórtico.

Se realizó electrocardiograma con elevación del ST en cara anterolateral, angiotomografía de tórax que descartó tromboembolia pulmonar y una curva de troponina positiva para isquemia. La ecocardiografía mostró hipocinesia difusa del ventrículo izquierdo, más acentuada hacia la pared inferoposterior con una fracción de eyección del ventrículo izquierdo del $20 \%$, ventrículo derecho normal con función sistólica moderadamente comprometida y TAPSE de $11 \mathrm{~mm}$. Fue llevada a coronariografía, que reveló placas no significativas del $40 \%$ en la descendente anterior y disminución del calibre de la arteria descendente anterior y la arteria circunfleja (fig. 1).

Con base en estos resultados se consideró como diagnóstico más probable una miocardiopatía periparto, para la cual se inició manejo, con el cual mejoró; fue dada de alta después de una estancia hospitalaria de 15 días.

A las 2 semanas consultó nuevamente al servicio de urgencias por disnea de pequeños esfuerzos y tos. Al ingreso se halló hipotensa, taquicárdica y con signos de hipoperfusión distal; durante la auscultación pulmonar se reconocieron crépitos generalizados. Se inició manejo para falla cardíaca crónica agudamente descompensada y se solicitó perfil de autoinmunidad los cuales se encontraron en rango de normalidad. A los 5 días de su ingreso tuvo un episodio de muerte súbita por fibrilación ventricular, para lo cual recibió maniobras de reanimación avanzada logrando retorno a circulación espontánea. Fue evaluada por Cardiología y Electrofisiología quienes consideraron que debía continuar su atención en un centro cardiovascular especializado en falla cardíaca para evaluar la posibilidad de un dispositivo de asistencia ventricular como terapia puente para un trasplante cardíaco.

Ingresó al tercer día a nuestra institución con presión arterial de $94 / 60 \mathrm{~mm} \mathrm{Hg}$, frecuencia cardíaca de 83 latidos por minuto, saturación al $98 \%$ sin oxígeno complementario, con soporte vasopresor con noradrenalina a dosis bajas, tolerando el decúbito.

Se revaluó la coronariografía inicial en la cual se confirmaron los hallazgos en las arterias epicárdicas, además de adelgazamiento de la arteria descendente anterior y circunfleja, y hallazgos que sugerían disección espontánea de las arterias coronarias izquierdas; sin embargo, no se contaba en la institución con ultrasonido intravascular o tomografía de coherencia óptica para confirmar esta sospecha diagnóstica. 


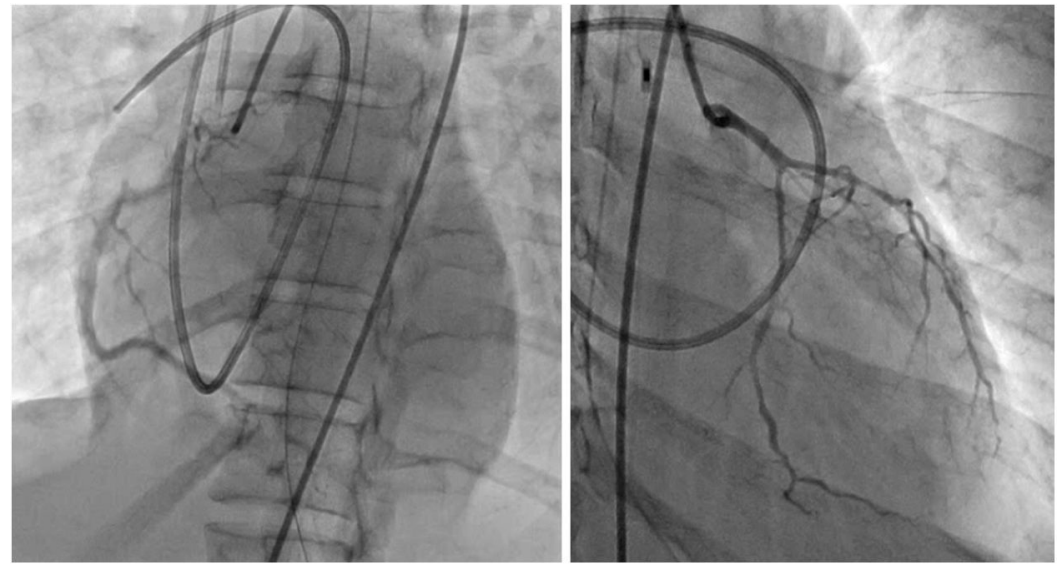

Figura 1 Coronariografía. A Arteria coronaria derecha. B Arteria coronaria izquierda.
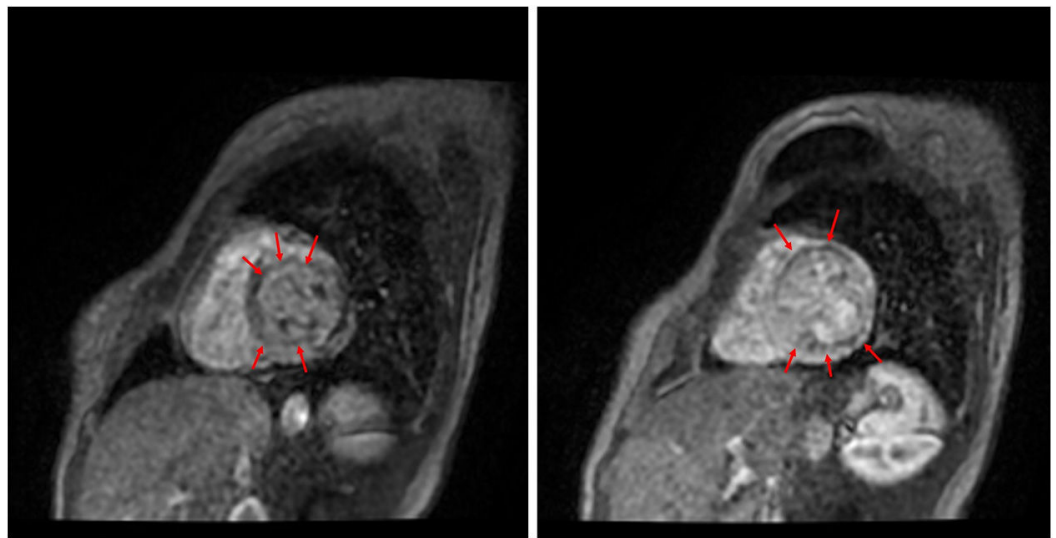

Figura 2 Resonancia cardíaca. A Defectos de perfusión simple. B Persistencia del defecto de perfusión en las imágenes de realce tardío.
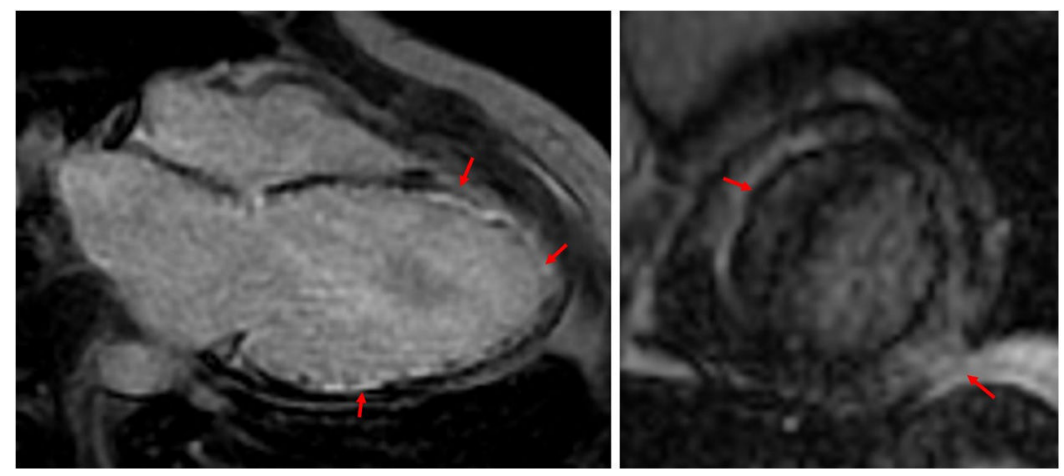

Figura 3 Realce tardío transmural en tercios medio y apical en territorio de la A. descendente anterior y A. circunfleja. A) Realce tardío 4 cámaras. B) Realce tardío eje corto.

Se realizó resonancia magnética cardiaca (figs. 2 y 3), en la cual la aurícula derecha tenía un tamaño normal y la aurícula izquierda dilatación moderada sin trombos o lesiones endoluminales. El ventrículo derecho era de tamaño normal, sin hipertrofia, función sistólica normal y TAPSE de $20 \mathrm{~mm}$. El ventrículo izquierdo presentaba gran dilatación sin hipertrofia por grosor de la pared e índice de masa y disfunción sistólica grave con fracción de eyección del $26 \%$. Adicionalmente, se observó adelgazamiento diastólico de los segmentos anterolateral e inferolateral en la región medio ventricular y los segmentos anterior y lateral del ápex, así como el ápex verdadero (segmentos 5,6,7,8,11,12,13,16 y 17). Estos segmentos presentaban hipocinesia grave y realce tardío transmural indicando infartos sin signos de viabilidad, con lo cual se descartó enfermedad infiltrativa o secuelas de miocarditis. Se evidenciaron compromisos extensos en territorio de las arterias descendente anterior y circunfleja e insuficiencia mitral leve a moderada secundaria a 
Tabla 1 Condiciones y factores asociados a la disección espontánea de arterias coronarias

\begin{tabular}{ll}
\hline Factores asociados & Factores precipitantes \\
\hline $\begin{array}{l}\text { Displasia fibromuscular } \\
\text { Embarazo }\end{array}$ & $\begin{array}{l}\text { Ejercicio intenso (isométrico o aeróbico) } \\
\text { Valsalva intensa. } \\
\text { Náuseas, vómito, movimientos intestinales, tos, } \\
\text { levantar objetos pesados. } \\
\text { Estrés emocional intenso. }\end{array}$ \\
$\begin{array}{l}\text { Arteriopatía heredada o enfermedad del tejido } \\
\text { conectivo: síndrome de Marfan, Síndrome de } \\
\begin{array}{l}\text { Loeys-Dietz, síndrome vascular de Ehlers-Danlos, } \\
\text { entre otros. }\end{array}\end{array}$ & $\begin{array}{l}\text { Trabajo de parto y parto. } \\
\text { Hormonas exógenas: anticonceptivos, tratamiento } \\
\text { para la infertilidad. } \\
\begin{array}{l}\text { Enfermedades inflamatorias sistémicas: lupus } \\
\text { eritematoso sistémico, colitis ulcerativa, }\end{array} \text { poliarteritis nodosa, sarcoidosis, poliangeítis con } \\
\text { granulomatosis eosinofílica, artritis reumatoide, } \\
\text { enfermedad de Kawasaki, enfermedad celíaca. }\end{array}$ \\
$\begin{array}{l}\text { Migraña. } \\
\text { Espasmo de arterias coronarias. }\end{array}$ & Drogas recreacionales (cocaína, metanfetaminas). \\
\hline $\begin{array}{l}\text { Adaptada de Hayes SN et al. Spontaneous Coronary Artery Dissection: Current State of the Science. } \\
\text { Inyecciones de B-HCG o corticosteroides. }\end{array}$
\end{tabular}

Adaptada de Hayes SN et al. Spontaneous Coronary Artery Dissection: Current State of the Science.

dilatación del anillo. En total se evidenciaron nueve segmentos comprometidos que indicaban un compromiso de aproximadamente el 50 al 55\% de la masa miocárdica total. Se consideró entonces que la causa de la cardiopatía isquémica no aterosclerótica de la paciente fue una disección espontánea de la arteria coronaria izquierda. Se continuó titulación del manejo de la falla cardíaca, pero presentó edema pulmonar con el inicio del betabloqueador y cifras tensionales en límite inferior que no permitían la dosis óptima de inhibidores de la enzima convertidora de angiotensina (IECA) ni de ivabradina y persistía con requerimiento de terapia diurética y milrinone. A raíz de esto, se inició protocolo para trasplante cardíaco. Durante la hospitalización, tuvo mejoría clínica y sintomática, toleró el ajuste del manejo médico por lo que fue posible dar de alta sin eventual requerimiento del trasplante, previo implante de desfibrilador para prevención secundaria.

\section{Discusión}

Una de las principales causas de mortalidad en el embarazo son las enfermedades de origen cardiovascular y de éstas el $20 \%$ se explica por un infarto agudo de miocardio. Las mujeres en estado de embarazo tienen tres a cuatro veces mayor riesgo que las no embarazadas de sufrir un infarto agudo de miocardio, en cuyo caso en el $25 \%$ se atribuye a disección espontánea de las arterias coronarias, mientras que en las mujeres no embarazadas ocurre en menos del $1 \%^{3}$.

La disección espontánea de las arterias coronarias es una entidad subdiagnosticada y potencialmente fatal, la cual se define como aquella en la que hay una separación entre las capas de la pared arterial, generalmente la íntima y la media, por un hematoma intramural que no es de origen traumático ni iatrogénico, ya sea debido a una laceración en la capa íntima o a una disrupción de la vasa vasorum $^{4,5}$.
La fisiopatología de la disección espontánea de las arterias coronarias no está completamente dilucidada; se han descrito múltiples factores que predisponen a ello. Los cambios hormonales durante el embarazo pueden provocar cambios estructurales en los vasos, tales como disminución de la síntesis del colágeno y aumento del contenido de mucopolisacáridos, y producir debilidad de la túnica media, asociada a los cambios en la fisiología cardiovascular, que aunados pueden aumentar el estrés en la pared de los vasos. En la tabla 1 se muestran los factores asociados y precipitantes $^{4,5}$.

La presentación clínica puede ir desde un estado completamente asintomático hasta la muerte súbita, según la gravedad y extensión de la disección ${ }^{6}$. Los marcadores bioquímicos y los signos electrocardiográficos son similares a los de un síndrome coronario agudo pero ocurre en mujeres jóvenes y en ausencia de los factores de riesgo cardiovascular típicos ${ }^{7}$.

El método diagnóstico inicial para el estudio de la disección espontánea de las arterias coronarias es la coronariografía; sin embargo, es difícil reconocer los múltiples lúmenes radiolúcidos con captación de contraste en la pared del vaso, que son el hallazgo patognomónico. Para mejorar el rendimiento diagnóstico se debe hacer un análisis con ecografía intravascular (IVUS) o una tomografía de coherencia óptica (OCT); no obstante, en la ecografía intravascular, en más del $70 \%$ de los casos no es posible observar con claridad la disección, de modo que se prefiere la tomografía de coherencia óptica pues ha mostrado ser superior para visualizar los hematomas intramurales. Pese a ello, es preciso tener precaución ya que requiere una presión alta de inyección del contraste, maniobra que puede aumentar el tamaño de la disección ${ }^{7-11}$.

En la coronariografía se pueden encontrar tres variantes de disección espontánea de las arterias coronarias que pueden complicar aún más el diagnóstico. La variante tipo 1 se caracteriza por doble lumen; es la más característica de la enfermedad, pero sólo se da en un tercio de los 


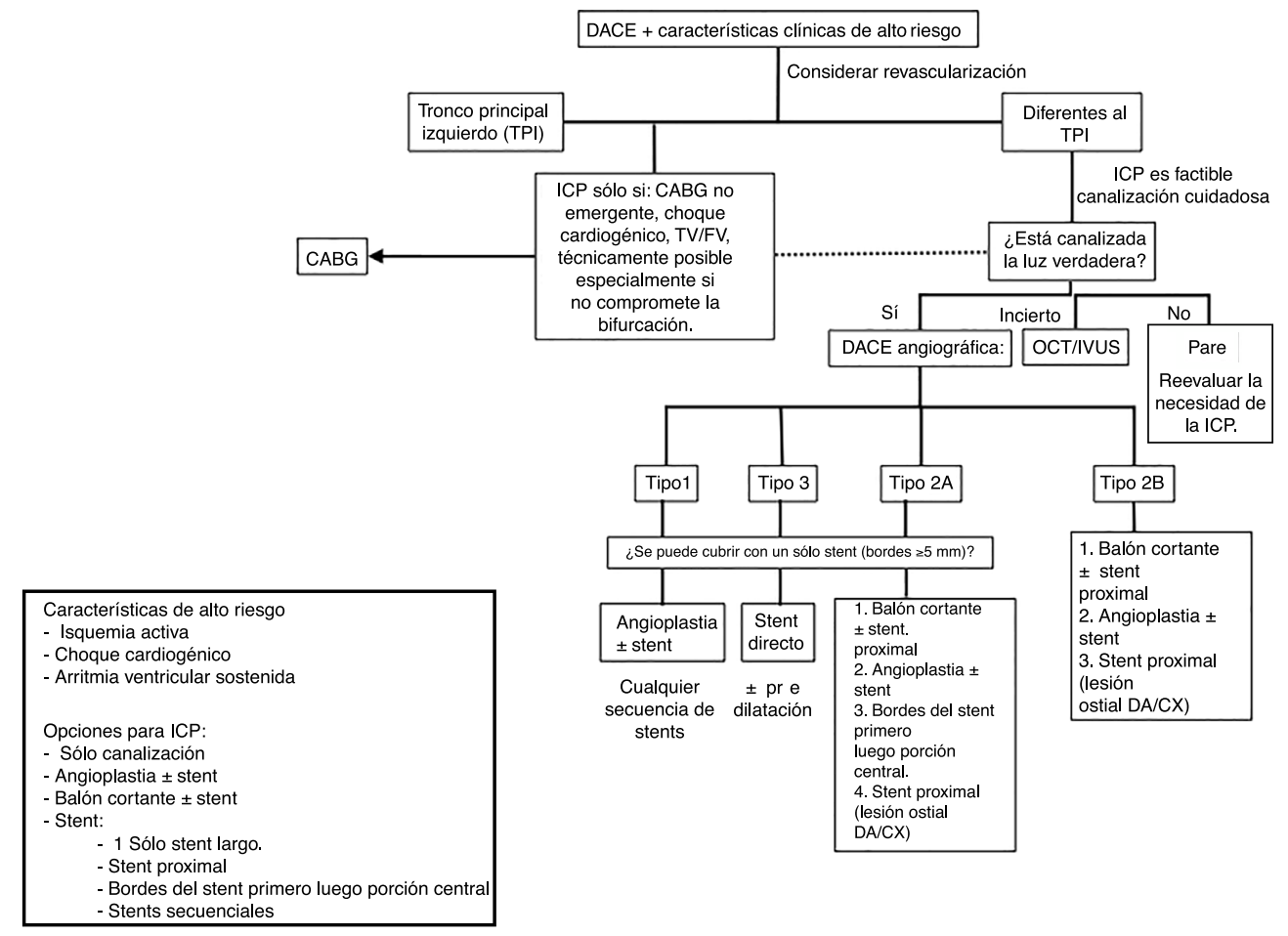

Figura 4 Algoritmo para el manejo de la disección espontánea de las arterias coronarias. DACE: Disección espontánea de arterias coronarias, CABG: Bypass coronario, TV: taquicardia ventricular, FV: fibrilación ventricular, OCT: tomografía de coherencia óptica, IVUS: ecografía intravascular, DA: arteria descendente anterior, CX: arteria circunfleja. Tomado con autorización de: Saw J. Natural history of spontaneous coronary artery dissection: to stent or not to stent? Eurointervention. 2019;14(13):1353-6, con permiso de Europa Digital \& Publishing.

pacientes. La variante tipo 2, que fue la que se evidenció en la paciente del caso que se expone, se caracteriza por estenosis difusa de rango de gravedad variable, con cambios en el calibre arterial; y la tipo 3, que asemeja una lesión aterosclerótica o trombótica. En general, las mujeres embarazadas tienen compromiso de la arteria coronaria izquierda a nivel proximal, pero también pueden presentar afectación de múltiples vasos y mayor compromiso de la función ventricular ${ }^{7,8,12}$.

En el estudio del infarto agudo de miocardio de etiología no clara, se debe ordenar una resonancia magnética cardíaca para evaluar las posibles etiologías y confirmar el compromiso isquémico miocárdico. En caso de que se identifique un área es preciso revisar nuevamente la coronariografía, como se hizo en el caso que se ilustra, en aras de identificar disección de las arterias coronarias o embolia a una rama pequeña y $\operatorname{distal}^{8}$.

Existe controversia respecto al tratamiento (fig. 4), si se debe realizar revascularización percutánea, quirúrgica o sólo manejo conservador. Como primera línea para la revascularización está la intervención coronaria percutánea siempre y cuando la anatomía sea favorable, excepto si hay compromiso del tronco principal izquierdo, disección proximal de más de dos vasos o en el caso de una intervención coronaria percutánea fallida en donde se prefiere la revascularización quirúrgica. La intervención coronaria percutánea se ha asociado con múltiples complicaciones periprocedimiento, ya que es técnicamente demandante introducir el catéter en el lumen verdadero, lo que explica una tasa de éxito de sólo el $65 \%$ e incluso hasta en el 25 al $60 \%$ de los casos se puede aumentar el tamaño de la disección. En caso que el paciente sea llevado a intervención coronaria percutánea se recomienda que el acceso sea vía femoral ya que en series retrospectivas se ha encontrado que por la vía radial se aumenta hasta tres veces el riesgo de realizar disecciones iatrogénicas ${ }^{6}$. En pacientes con estabilidad hemodinámica y flujo TIMI 2 o 3 se prefiere el manejo conservador; el $100 \%$ de de los casos tendrán resolución de la disección el día 26 posterior al evento $^{12,13}$.

El tratamiento médico es similar al del infarto agudo de miocardio de origen aterosclerótico. Se recomienda utilizar ácido acetilsalicílico como prevención secundaria por al menos un año y, desde que no haya contraindicaciones, toda la vida. En cuanto a la adición de clopidogrel, si bien se ha considerado que el uso dual de estos antiplaquetarios podría disminuir el riesgo de trombosis del falso lumen y la compresión consecuente del lumen verdadero, no se recomienda utilizar esta combinación excepto en los casos en los que se haya implantado un stent ya que no se ha demostrado un beneficio claro ${ }^{14}$.

Se debe considerar el uso de betabloqueadores con el fin de disminuir el estrés de la pared y el riesgo de disección recurrente de las arterias coronarias. Los IECA se 
usan en caso de disfunción ventricular de acuerdo con las guías de síndrome coronario agudo. El empleo de las estatinas en infarto agudo de miocardio no aterosclerótico no se ha estudiado e incluso en algunas series retrospectivas se ha mostrado asociación entre el uso de estatinas y mayor riesgo de recurrencia de un nuevo evento, de ahí que se aconseje utilizar sólo en el paciente con dislipidemia concomitante. No es clara la utilidad de la anticoagulación para disolver el trombo de la falsa luz. Se utilizan las heparinas en el momento inicial; si después de la coronariografía se confirma disección espontánea de las arterias coronarias, se debe suspender por el riesgo de aumentar la extensión del hematoma intramural. No se debe realizar trombólisis ya que aumenta la extensión de la disección y el tamaño del hematoma intramural ni tampoco utilizar inhibidores de glicoproteína IIb/IIla2,6,7,12,14.

La mortalidad intrahospitalaria es del 1 al $5 \%$, con una posibilidad de recurrencia en el $22 \%$ de los casos, ocurre principalmente en los vasos que no habían sido afectados y 30 días o más después del primer evento; hasta ahora el único factor de riesgo identificado es la tortuosidad de la arteria coronaria. El pronóstico de la disección espontánea de las arterias coronarias en mujeres embarazadas es peor, ya que se encuentran más enfermas en el momento del suceso, presentan infartos más grandes y hay mayor compromiso de la función del ventrículo izquierdo, lo que implica mayor incidencia de choque cardiogénico y de falla cardíaca congestiva ${ }^{7}$.

\section{Conflictos de interés}

Ninguno.

\section{Bibliografía}

1. Dubbs SB, Tewelde SZ. Cardiovascular catastrophes in the obstetric population. Emerg Med Clin North Am. 2015;33: 483-500.

2. Yip A, Saw J. Spontaneous coronary artery dissection-A review. Cardiovasc Diagn Ther. 2015;5:37-48.
3. Moussa H, Movahedian M, Leon M, Sibai B. Acute Myocardial Infarction Due to Coronary Artery Dissection in the Postpartum Period. Am J Perinatol Rep. 2015;05:e093-96.

4. Saw J, Humphries K, Aymong E, Sedlak T, Prakash R, Starovoytov A, et al. Spontaneous coronary artery dissection. J Am Coll Cardiol. 2017;70:1148-58.

5. Rose E, Gedela M, Miller N, Carpenter PL. Pregnancy-related spontaneous coronary artery dissection: a case series and literature review. J Emerg Med. 2017;52:867-74.

6. Shah SR, Alweis R. Acute coronary artery dissection: a review of the literature and current evidence. Cardiol Rev. 2018;26:274-6.

7. Vandamme M, De Backer J, De Backer T, Drieghe B, Devos D, Gevaert S. The spectrum of spontaneous coronary artery dissection: illustrated review of the literature. Acta Cardiol. 2017;72:599-609.

8. Lebrun S, Bond RM. Spontaneous coronary artery dissection (SCAD): The underdiagnosed cardiac condition that plagues women. Trends Cardiovasc Med [Internet]. 2017 [citado 25 de febrero de 2018]; Disponible en: http://linkinghub. elsevier.com/retrieve/pii/S1050173817301925.

9. Luong C, Starovoytov A, Heydari M, Sedlak T, Aymong E, Saw J. Clinical presentation of patients with spontaneous coronary artery dissection: Clinical Presentation of Patients with SCAD. Catheter Cardiovasc Interv. 2017;89:1149-54.

10. Buccheri D, Milazzo D, Geraci S, Vaccaro G, Caramanno G. A lesson from intravascular imaging: insights for recognizing a spontaneous coronary artery dissection. J Thorac Dis. 2017;9:5363-7.

11. Pierce T, Hovnanian M, Hedgire S, Ghoshhajra B. Imaging of cardiovascular disease in pregnancy and the peripartum period. Curr Treat Options Cardiovasc Med [Internet]. 2017 [citado 25 de febrero de 2018];19(12). Disponible en: http://link.springer.com/10.1007/s11936-017-0593-8.

12. Jneid H. Spontaneous Coronary Artery Dissection. JACC Cardiovasc Interv. 2018;11:91-4.

13. Saw J. Natural history of spontaneous coronary artery dissection: to stent or not to stent? Eurolntervention. 2019;14:1353-6.

14. Hayes SN, Kim ESH, Saw J, Adlam D, Arslanian-Engoren C, Economy KE, et al. Spontaneous Coronary Artery Dissection: Current State of the Science: A Scientific Statement From the American Heart Association. Circulation. 2018:CIR, 0000000000000564. 